\title{
Abnormalities in circulating plasmacytoid dendritic cells in patients with systemic lupus erythematosus
}

\author{
Ou Jin1,2, Sushma Kavikondala1', Mo-Yin Mok1, Lingyun Sun³, Jieruo Gu², Rong Fu'1, Albert Chan1', Joseph Yeung1, \\ Yingjie Nie 1 and Chak-Sing Lau*1
}

\begin{abstract}
Introduction: Dendritic cells (DCs) are capable of inducing immunity or tolerance. Previous studies have suggested plasmacytoid DCs (pDCs) are pathogenic in systemic lupus erythematosus (SLE). However, the functional characteristics of directly isolated peripheral circulating blood pDCs in SLE have not been evaluated previously.

Methods: Peripheral blood pDCs from 62 healthy subjects and 58 SLE patients were treated with apoptotic cells derived from polymorphonuclear cells (PMNs). Antigen loaded or unloaded pDCs were then co-cultured with autologous or allogenous T cells. Changes in T cell proliferation, cell surface CD25 expression, intracellular Foxp3 expression and cytokine production were evaluated. pDCs that had captured apoptotic PMNs (pDCs + apoPMNs were also studied for their cytokine production (interferon (IFN)-alpha, interleukin (IL)-6, IL-10, IL-18) and toll like receptor (TLR) expression.

Results: Circulating pDCs from SLE patients had an increased ability to stimulate T cells when compared with control pDCs. Using allogenous T cells as responder cells, SLE pDCs induced T cell proliferation even in the absence of apoptotic PMNs. In addition, healthy pDCs + apoPMNs induced suppressive T regulatory cell features with increased Foxp3 expression in CD4 + CD25 + cells while SLE pDCs + apoPMNs did not. There were differences in the cytokine profile of pDCs that had captured apoptotic PMNs between healthy subjects and patients with SLE. Healthy pDCs + apoPMNs showed decreased production of IL-6 but no significant changes in IL-10 and IL-18. These pDCs + apoPMNs also showed increased mRNA transcription of TLR9. On the other hand, while SLE pDCs + apoPMNs also had decreased IL-6, there was decreased IL-18 mRNA expression and persistent IL-10 protein synthesis. In addition, SLE pDCs lacked TLR9 recruitment.

Conclusions: We have demonstrated that peripheral circulating pDCs in patients with SLE were functionally abnormal. They lacked TLR9 expression, were less capable of inducing regulatory T cell differentiation and had persistent IL-10 mRNA expression following the capture of apoptotic PMNs. We suggest circulating pDCs may be pathogenically relevant in SLE.
\end{abstract}

\section{Introduction}

Dendritic cells (DCs) are professional antigen presenting cells (APCs) with important immunoregulatory functions. They are the only cells that can stimulate naïve $\mathrm{T}$ cells [1]. Commonly, DCs circulate in peripheral tissues, capture pathogens or dying cells, and present antigens to

* Correspondence: cslau@hkucc.hku.hk

${ }^{1}$ Division of Rheumatology and Clinical Immunology, Department of Medicine, Queen Mary Hospital, The University of Hong Kong, 102 Pokfulam Road, Hong Kong, PR China

Full list of author information is available at the end of the article
$\mathrm{T}$ cells. $\mathrm{T}$ cells then proliferate and differentiate into Th1 (cell immunity), Th2 (humoral immunity) or T regulatory (Treg) (suppressive) cells resulting in the induction of immunity or tolerance [2]. Recent studies have shown that cytokines produced by DCs contribute to the induction of $\mathrm{T}$ cell differentiation [1]. For example, interleukin (IL) -6 primes $\mathrm{CD} 4{ }^{+} \mathrm{T}$ cells to differentiate into Th2 cells [3] and suppresses the activity of Treg cells [4]. IL-10 inhibits Th1 but induces Th2 responses [5]. IL-18, previously known as IFN- $\gamma$-inducing factor, interacts with IL- 
12 and induces naive $\mathrm{T}$ cell proliferation and differentiation into interferon (IFN)- $\gamma$ producing Th1 cells [6].

Considering the role of DCs in the induction of immunity or tolerance in health, changes in DC function in autoimmune diseases such as systemic lupus erythematosus (SLE) which is characterized by loss of tolerance to self antigens have been studied intensively. Actually, DC functional abnormalities in SLE have been reported previously, the main progress of which is on the pathogenic role of plasmacytoid DCs (pDCs) in this condition [7]. Though the precise role of pDCs in the immune system is still unclear, these cells have been shown to polarize $T$ cells through its high production of IFN- $\alpha$ [2], which is generally considered to be the central cytokine that contributes to SLE development [8]. However, it is puzzling that some previous studies have reported diminished IFN- $\alpha$ production and $\mathrm{T}$ cell-stimulatory capacity by cytokine-induced pDCs in SLE [9]. Further, high serum levels of IFN- $\alpha$ are only found in some but not all SLE patients [10]. The role of pDCs in SLE requires further clarification.

Apoptotic cells are the primary source of autoantigens in SLE $[11,12]$. Nucleic-acid containing macromolecules which comprise the majority of autoantigens in SLE have been detected on the surface of apoptotic bodies [13]. The hypothesis is that in the process of apoptosis, nuclear antigens are cleaved into DNA fragments, revealing previously cryptic epitopes or neoepitopes to activate the immune system [12]. Supportive evidence comes from studies which showed DNA fragments isolated from sera of SLE patients stimulated mononuclear cell proliferation, and that these DNA fragments were of the same size as internucleosomal digested DNA from apoptotic cells [14].

Recently, an animal study has shown apoptotic cellpulsed bone marrow-derived DCs (AC-BMDCs) could induce the proliferation of self reactive $\mathrm{T}$-cells resulting in tolerance break down and initiation of autoimmune responses in normal mice [15]. However, until now no direct studies have been carried out in human SLE evaluating the function of peripheral blood pDCs and their interactions with immune cells following loading with apoptotic cells. In this study, we carried out experiments to examine the characteristics and functions of freshly isolated circulating pDCs from healthy and SLE subjects in the absence or presence of apoptotic cells. We hypothesize that peripheral circulating pDCs are functionally abnormal in SLE.

\section{Materials and methods Subjects}

Patients who fulfilled the American College of Rheumatology criteria for SLE [16] were studied. There were 50 females and 8 males, with their age ranging from 21 to 62
$(41.76 \pm 9.15)$ years and disease duration from 1 to 28 $(10.58 \pm 7.16)$ years. Disease activity was assessed using the SLE disease activity index (SLEDAI) [17]. Active disease was defined as SLEDAI $\geq 5(n=26)$. Thirty-two patients had inactive disease (SLEDAI $<5)$. Sixty-two sexand age-matched healthy volunteers were recruited from the Red Cross Blood Transfusion Section. The study was approved by the Hong Kong West Cluster Institutional Review Board for medical ethics. All subjects provided a written informed consent.

\section{An overview of the experimental design}

(1) Circulating blood pDCs were isolated and cultured with or without apoptotic cells; (2) Antigen loaded or unloaded pDCs were then co-cultured with autologous or allogenous $\mathrm{T}$ cells. The same control $\mathrm{T}$ cells were used as responder cells in all allogenic proliferation assays. Changes in T cell proliferation, cell surface marker CD25 expression, $\mathrm{CD} 4{ }^{+} \mathrm{CD} 25^{+}$Foxp $3{ }^{+}$expression and cytokine production were evaluated; and (3) functional changes of pDCs after interaction with apoptotic cells were evaluated by detection of cytokine production and toll-like receptor (TLR) expression.

\section{Blood collection and cell isolation}

A total of $100 \mathrm{mls}$ of sodium citrate anti-coagulated blood were collected between 9:00 and 11:00 AM. Peripheral blood mononuclear cells (PBMCs) were freshly isolated by Ficoll density gradient centrifugation. pDCs and $\mathrm{T}$ cells from PBMCs were magnetically sorted with the human BDCA-4 DC and pan T isolation kits respectively according to the manufacturer's description (Miltenyi Biotec, Berqisch Gladbach, Germany). Briefly, pDCs were positively selected using anti-BDCA-4 conjugated beads [18]. $T$ cells were negatively isolated using magnetic beads conjugated with various surface antigen antibodies (anti-CD14, anti-CD16, anti-CD19, anti-CD36, antiCD56, anti-CD123) to remove non-T cells. The purity of pDCs and T cells was approximately $90 \%$ and approximately $99 \%$, respectively.

After isolating PBMCs from the whole blood, dextran sedimentation was applied to separate polymorphonuclear cells (PMNs, neutrophils) from red blood cells (RBCs). The remaining RBCs were lyzed with ammonium chloride (BD, $\mathrm{CA}$ ) and removed by washing with saline. Purified PMNs were then resuspended with complete RPMI 1640 medium at a concentration of $5 \times 10^{6} \mathrm{cells} / \mathrm{ml}$. PMNs were given $120 \mathrm{~mJ} / \mathrm{ml}$ ultraviolet (UV) irradiation using the CL-100 Ultraviolet Crosslinker (Upland, CA, USA) to induce apoptosis. Sixteen hours after UV irradiation, the rate of apoptosis on PMNs reached around 60\% to $90 \%$ and was confirmed by microscopic examination of cytocentrifuge stained with May-Giemsa, and flow cyto- 
metric detection of annexin $\mathrm{V}$ and propidium iodide (PI) staining (double positive cells).

\section{Interaction of dendritic cells with apoptotic cells}

pDCs were incubated with apoptotic PMNs for four hours. pDCs that had captured apoptotic PMNs (pDCs + apoPMNs) were confirmed by flow cytometry detection of surface PE-Cy5 CD123 (for pDC) and 5-(and 6)-carboxytetramethylrhodamine succinimidyl ester (for PMNs) double positive stained cells, the percentage of which was around $15 \%$. pDCs or pDCs + apoPMNs were then treated with mitomycin $\mathrm{C}$, which has the ability to inhibit proliferation without affecting the viability of the feeder cells. The cell cultures were subsequently washed with phosphate buffer solution (0.5\% BSA) to remove mytomycin $\mathrm{C}$ and apoptotic PMNs, for T cell interaction experiments or for evaluation of cytokine and TLR expression.

\section{Mixed leukocyte reaction (MLR)}

$\mathrm{T}$ cell proliferation and differentiation induced by $\mathrm{pDCs}$ were evaluated by MLR. In brief, pDCs $\left(1.0 \times 10^{4}\right)$ or pDCs that had interacted with apoptotic PMNs for four hours (pDC:PMN ratios = 1:1, 1:5, and 1:10) were used to stimulate autologous or allogenous responder T cells (1.0 $\times 10^{5}$ ). After five days of culture in a 96-well round-bottom culture plates with $200 \mu \mathrm{l}$ of complete RPMI 1640 culture medium, the culture supernatants were collected for the detection of cytokines produced by $\mathrm{T}$ cells, and fresh medium was added back. Following that, $0.5 \mu \mathrm{Ci}$ tritiated thymidine (Radiochemical Centre, Amersham, Little Chalfont, Buckinghamshire, UK) was added per well for 16 hours. $\mathrm{T}$ cell proliferation was measured by detection of the incorporation of $(3 \mathrm{H})$-thymidine. Cells were harvested with a Packard FilterMate ${ }^{\mathrm{m} x}$ Universal Harvester (Downers Grove, IL, USA) and read with a TopCount ${ }^{\text {tw }}$ NXT Microplate Scintillation Counter (Perkin Elmer, Boston, MA, USA). After the initial experiments, we found the 1:10 pDC:PMN ratio resulted in maximum $\mathrm{T}$ cell stimulation. This ratio was used in later experiments.

\section{Flow cytometry}

The purity of pDCs and $\mathrm{T}$ cells was confirmed by surface immunofluorescence staining and flow cytometry analysis. pDCs were defined as BDCA-2+CD123+lin- cells [19] and its purification was above 90\% [18]. Comparable levels of surface markers expression including MHC-II CD86 CD83 and CCR7 were found in pDCs from both SLE and healthy [18]. T cells were defined as $\mathrm{CD}^{+}$cells and its purification is about 99\% (data not shown). Changes in surface CD25 expression of T cells stimulated by pDCs were measured by percentage of $\mathrm{CD} 4{ }^{+} \mathrm{CD} 25^{+}$ cells per $\mathrm{CD}^{+} \mathrm{T}$ cells and compared with that of unstim- ulated $\mathrm{T}$ cells. To further investigate whether the $\mathrm{CD} 4{ }^{+} \mathrm{CD} 25^{+} \mathrm{T}$ cells were Treg cells or not, intracellular immunofluorescence staining of Foxp3 was carried out according to the manufacturer's description (eBioscience, San Diego, CA, USA). Appropriate isotype-matched control Ig was used as negative controls for each analysis. Flow cytometric analysis was performed within 24 hours, on a FACSCalibur flow cytometry using Cellquest software.

\section{ELISA}

Cytokines produced by $\mathrm{T}$ cells after interaction with pDCs or pDCs + apoPMNs were measured using OptEIATM ELISA (BD Biosciences Pharmingen, San Diego, CA, USA). Supernatants in the MLR system were collected after five days culture and frozen at $-70^{\circ} \mathrm{C}$ for subsequent cytokine detection. These cytokines included IFN- $\gamma$, tumor necrosis factor (TNF)- $\alpha$ and IL-2 (Th1 cytokines); IL-4 and IL-6 (Th2 cytokines); and IL-10 and transfer growth factor (TGF) $\beta$ (Treg cytokines) [2].

Changes in cytokine production by pDCs or pDCs + apoPMNs were evaluated also using ELISA. Supernatants from the pDC culture system were collected after 24 hours' culture and frozen at $-70^{\circ} \mathrm{C}$ for subsequent detection of IFN- $\alpha$, IL-6, IL-10, IL-12, TNF- $\alpha$ levels. Human IFN- $\alpha$ ELISA kit was obtained from PBL Biomedical Laboratory (Piscataway, NJ, USA). ELISA results were read using MQX200 FCC Compliance (BID-TEK Instruments, Inc., Sacramento, CA, USA).

\section{Real time polymerase chair reaction (PCR)}

Semi-quantitative real time PCR was applied to detect changes in cytokine or TLR mRNA gene expression in pDCs after interaction with apoptotic cells for four hours. pDCs without interaction with apoptotic PMNs and PMNs alone were used as controls. Total RNA from the culture system was isolated through PurelinkTM Microto-Midi Total RNA Purification System (Invitrogen, Carlsbad, CA, USA) according to the manufacturer's description. First-strand complementary DNA was subsequently synthesized using the SuperScriptTM FirstStand Synthesis System (Invitrogen). For real-time PCR detection of target and housekeeping gene expression, the fluorescent TaqMan 5'-nuclease assay was performed using $2 \times$ TaqMan Universal PCR Master Mix and TaqMan Gene Expression assays (two unlabeled primers and one 6-FAM or VIC dye -labeled TaqMan MGB probe) for IL-6 (Hs00174131-ml), IL-10 (Hs00174086-ml), IL-18 (Hs00155517-ml), IFN- $\alpha 1$ (Hs00256882-sl), TLR-4 (Hs00152939-ml), TLR-7 (Hs00152971-ml), TLR-9 (Hs00370913-sl) and 18S (Hs999999901-sl) (Applied Biosystems, Foster City, CA, USA). The reaction was performed in triplicate on an ABI 7000 Sequence Detector (Applied Biosystems) with a standard run. The expression 
levels of the target genes were adjusted to that of the housekeeping genes 18S. The fold change in the pDC target gene expression was adjusted to that of 0 hour, represented as the 2- $-\Delta \Delta \mathrm{Ct}$, where $\Delta \Delta \mathrm{Ct}=$ (Ct.target Ct.18S $)_{\text {Time.x }}-(\text { Ct.target }- \text { Ct.18S })_{\text {Time.0h }}$.

\section{Statistical analysis}

As this was the first study on human peripheral circulating pDCs, the data were first tested for normality before statistical analysis to detect group differences was carried out. Normally distributed data are expressed as mean \pm $\mathrm{SD}$ while non-normally distributed data as median (interquartile range). For comparison across different groups, a two-factor analysis of variance (ANOVA) was used for normally distributed data. In case the ANOVA indicated differences across groups $(P<0.10)$, a pair-wise Student's $t$-test was used to evaluate the explicit $P$-values. Similarly, the Kruskal-Wallis test was used to compare across different groups of non-normally distributed data. If differences were indicated across groups, a Wilcoxon's rank sum test for two samples was used to evaluate the explicit $P$-values.

\section{Results}

T cell proliferation stimulated by $\mathrm{pDCs}$ or $\mathrm{pDCs}+$ apoPMNs Autologous MLR T cell proliferation

pDCs from healthy donors $(n=36)$ did not induce autologous $\mathrm{T}$ cell proliferation whether they were fed with apoptotic cells or not (Figure 1a). Similarly, SLE $(n=30)$ pDCs did not stimulate autologous $\mathrm{T}$ cell proliferation, irrespective of the overall SLE disease activity (Figure 1a). Allogenous MLR T cell proliferation

In the allogenous MLR system, only pDCs + apoPMNs from healthy subjects $(n=36)$ induced allogenous T cell proliferation while both $\mathrm{pDCs}$ and $\mathrm{pDCs}+$ apoPMNs from SLE patients $(n=30)$ were able to induce T cell proliferation (Figure 1b). As pDCs have a high rate of apoptosis themselves [20] (approximately 40\%, unpublished data), pDCs alone cultures may be regarded as a system of low level self-antigen presentation. These results suggest SLE pDCs have a higher ability to stimulate $T$ cells than controls. This was observed irrespective of the patients' disease activity.

\section{CD25 and Foxp3 expression on CD4 ${ }^{+} \mathrm{T}$ cells}

In the autologous MLR system, neither healthy nor SLE pDCs or pDCs + apoPMNs increased CD25 expression on $\mathrm{CD} 4{ }^{+} \mathrm{T}$ cells (Figure 2a). In the allogenous MLR system, both healthy and SLE pDCs + apoPMNs induced CD25 expression on CD4+ $\mathrm{T}$ cells (Figure 2b). Consistent with the proliferation assay results, SLE pDCs alone increased CD25 expression on allogenous $\mathrm{CD} 4{ }^{+} \mathrm{T}$ cells (Figure 2b), indicating that SLE pDCs had a higher ability to stimulate $\mathrm{CD} 25$ expression on allogenous $\mathrm{CD} 4{ }^{+} \mathrm{T}$ cells. (a) T cell Proliferation in Autologous MLR system

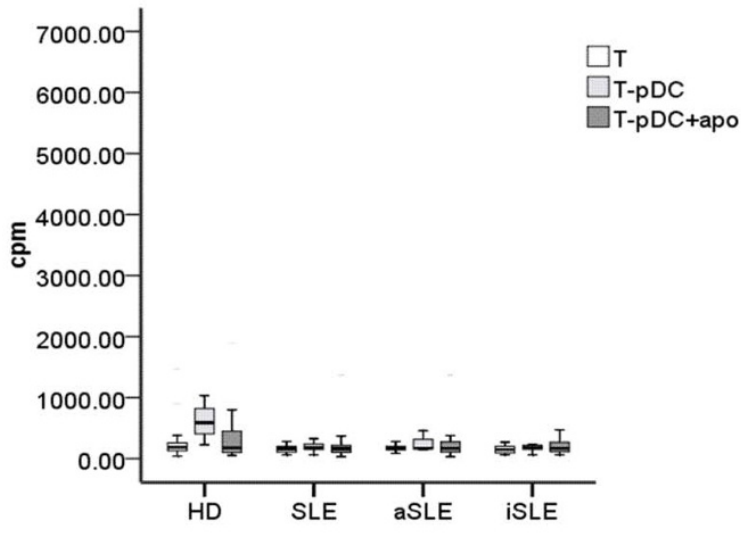

(b) T cell Proliferation in Allogenous MLR System

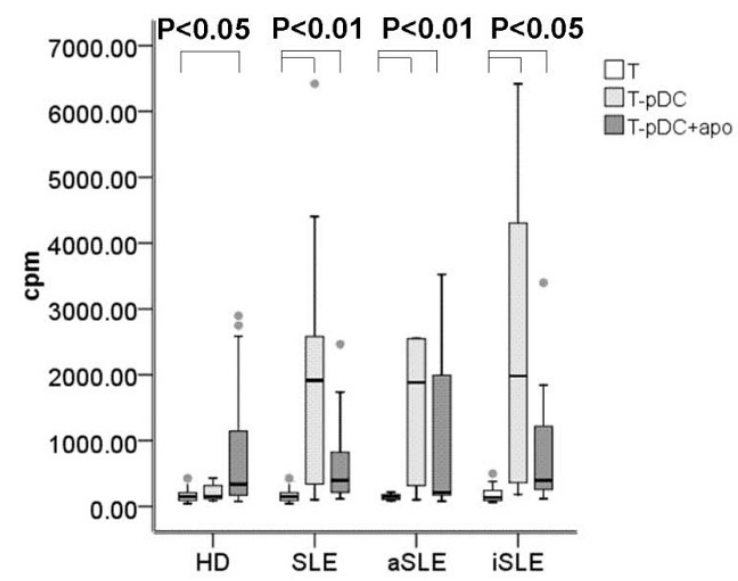

Figure $1 \mathrm{~T}$ cell proliferation following stimulation with $\mathrm{pDCs}$ alone or pDCs + apoPMNs in the MLR systems. (a) In the autologous MLR system: pDCs from healthy donors $(n=36)$ did not induce T cell proliferation, regardless of loading with apoptotic PMNs or not. The same phenomenon was also found in SLE patients $(n=30 ; 12$ with active disease and 18 with inactive disease), irrespective of disease activity status. (b) In the allogenous MLR system: In healthy donors ( $n=$ 36), only pDCs + apoPMNs induced allogenous T cell proliferation. With SLE pDCs, both pDCs alone and pDCs + apoPMNs induced allogenous T cell proliferation, suggesting a higher ability of SLE pDCs to stimulate allogenous T cells. Values are expressed in Median (interquartile range, range). auto-T: autologous T cells, allo-T: allogenous T cells, pDCs + apoPMNs: pDCs that had been loaded with apoptotic polymorphonuclear cells. HD, healthy donors; aSLE, active SLE; iSLE, inactive SLE

To evaluate whether the $\mathrm{CD} 25^{+} \mathrm{CD} 44^{+} \mathrm{T}$ cells may represent either active effector $\mathrm{T}$ cells or immunosuppressive Treg cells [21], intracellular staining and flow cytometry detection of Foxp3, Treg cell signatory, [22] were carried out. The percentage of $\mathrm{CD} 4{ }^{+} \mathrm{CD} 25^{+} \mathrm{T}$ cells that were Foxp $3^{+}$was evaluated. Neither healthy nor SLE pDCs changed the expression of Foxp3 in autologous 


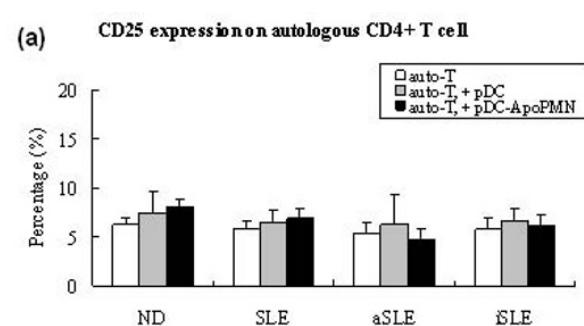

(b) CD25 expression on allogenous $\mathrm{CD} 4+\mathrm{T}$ cell

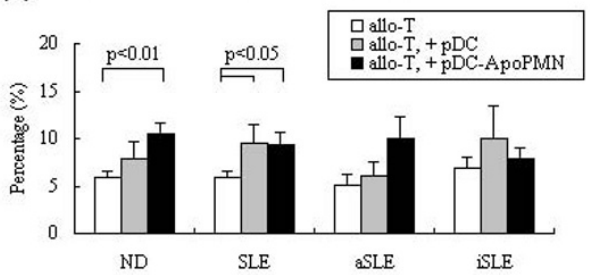

(c) Foxp3 on autologous CD4+CD25+ T cell

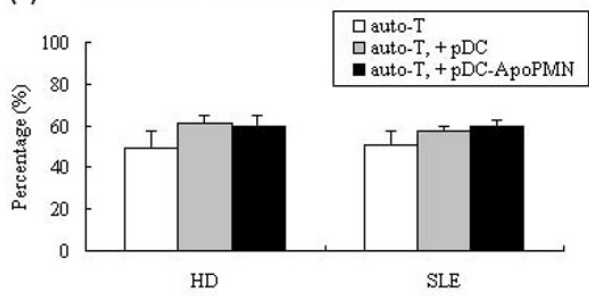

(d) Foxp3 on allogenous CD4+CD25 $+\mathrm{T}$ cell

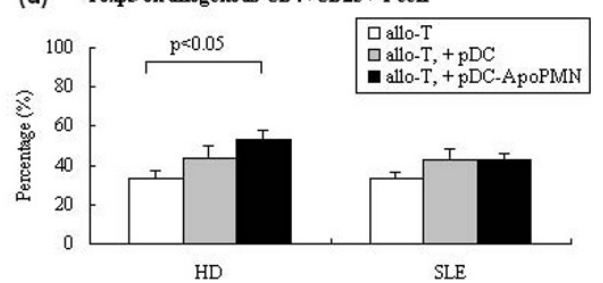

Figure 2 CD4 + T cell CD25 and Foxp3 expression following incubation with pDCs or pDCs + apoPMNs in MLR systems. (a) Autologous MLR system T cell CD25 expression: No changes were detected on CD4 + T cells from either healthy subjects $(n=36)$ or patients with SLE ( $n=30 ; 12$ with active disease and 18 with inactive disease) following incubation with $\mathrm{pDC}$ alone or pDCs + apoPMNs. Values are expressed in Median (interquartile range, range). (b) Allogenous MLR system T cell CD25 expression: CD25 expression was increased following incubation with healthy pDCs + apoPMNs $(n=36)$ but not pDCs alone, whereas a significant increase was seen following incubation with both SLE pDCs alone and pDCs + apoPMNs $(n=30)$. Values are expressed in Median (interquartile range, range). (c) Autologous MLR system CD4 + CD25 + T cell intracellular staining of Foxp3: No changes were detected following incubation with either $\mathrm{pDC}$ alone or $\mathrm{pDCs}+$ poPMNs from healthy subjects $(n=6)$ and patients with SLE $(n=6)$. Values are expressed in Mean \pm SEM. (d) Allogenous MLR system CD4 + CD25 + T cell intracellular staining of Foxp3: pDCs + apoPMNs from healthy donors increased the expression of Foxp3 on allogenous CD4 + CD25 + T cells $(n=8, P<0.05)$, but no significant changes were seen with SLE pDCs alone or pDCs + apoPMNs $(n=8)$. Values are expressed in Mean \pm SEM. auto-T: autologous T cells, allo-T: allogenous T cells, pDCs + apoPMNs: pDCs that had been loaded with apoptotic polymorphonuclear cells. HD, healthy donors; aSLE, active SLE; iSLE, inactive SLE.
$\mathrm{CD} 4{ }^{+} \mathrm{CD} 25^{+} \mathrm{T}$ cells (Figure 2c). With allogenous $\mathrm{CD} 4{ }^{+} \mathrm{CD} 25^{+} \mathrm{T}$ cells, however, healthy pDCs + apoPMNs increased the expression of Foxp3 (Figure 2d, $P<0.05$ ) while no significant changes were found with either SLE pDCs alone or pDCs + apoPMNs. These results suggest that healthy but not SLE pDCs induced the development of suppressive Treg cells.

\section{T cell cytokine production}

Neither healthy nor SLE pDCs or pDCs + apoPMNs induced autologous $\mathrm{T}$ cells to produce Th1 (Figure 3a1, Figure $3 \mathrm{~b} 1$ ) or Th2 related cytokines (data not shown). In the allogenous MLR system, both healthy and SLE pDCs + apoPMNs induced Th1 cell related cytokines including IFN $\gamma$ and IL-2 (Figure 3a2, 3b2).

Both autologous and allogenous $\mathrm{T}$ cells that were cocultured with healthy pDCs produced a small amount of Treg cell related TGF $\beta$ (Figure 3c) but the changes were not statistically significant.

\section{Changes in the functional characteristics of pDCs + apoPMNs \\ pDC cytokine production}

Following incubation with apoptotic cells for four hours, both healthy and SLE pDCs showed a slight increase in the expression of IFN $\alpha$ mRNA but it did not reach statistical significance (Figure 4a1). Both healthy and SLE pDCs + apoPMNs had decreased mRNA expression of IL-6 (Figure 4a2). There were no significant changes in the IL-10 mRNA expression of both healthy and SLE pDCs + apoPMNs (Figure 4a3). IL-18 mRNA expression in normal pDCs + apoPMNs did not change significantly but SLE pDCs - apoPMNs had decreased IL-18 mRNA expression (Figure 4a4).

Results of the ELISA of cytokines in supernatants of pDCs + apoPMNs cultures were in accordance with mRNA expression findings above. There were no changes in the level of IFN $\alpha$ from healthy and SLE pDCs + apoPMNs (Figure 4b1). IL-6 production by healthy and SLE pDCs + apoPMNs was both decreased (Figure 4b2). Interestingly, however, IL-10 production by healthy $\mathrm{pDCs}$ + apoPMNs was reduced but remained unchanged in SLE pDCs + apoPMNs (Figure 4b3).

\section{pDC TLR expression}

TLR9 mRNA expression in healthy pDCs + apoPMNs was found to be increased (Figure 5c) $(P<0.05)$. This was not observed with SLE pDCs + apoPMNs. No significant differences in the changes in TLR7 and TLR4 mRNA expression in between healthy and SLE pDCs + apoPMNs were detected (Figure 5a, b).

\section{Discussion}

Previous reports have shown pDCs induce immune tolerance after phagocyting apoptotic cells [23]. However, 


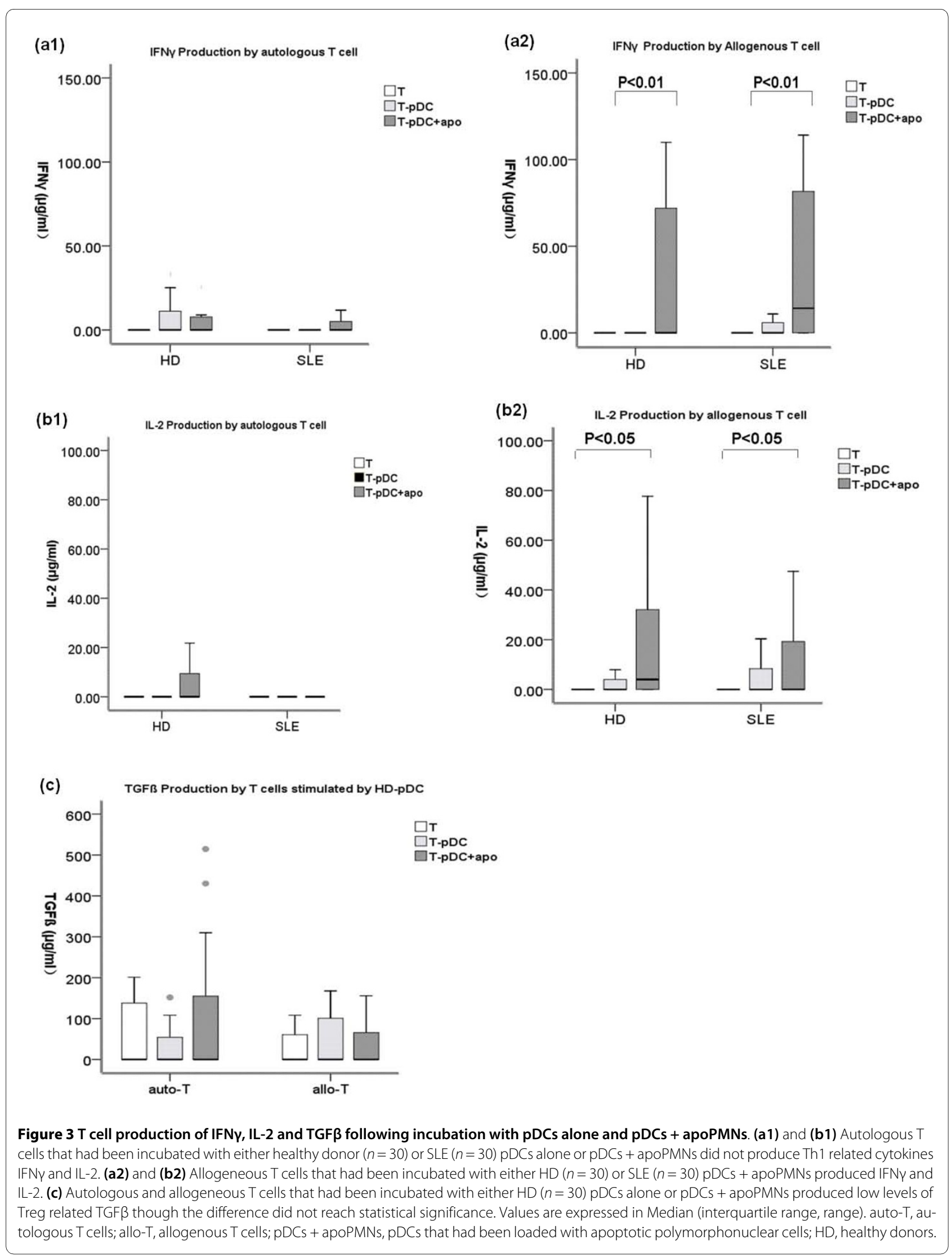


Lin et al. Arthritis Research \& Therapy 2010, 12:R137

Page 7 of 11

http://arthritis-research.com/content/12/4/R137
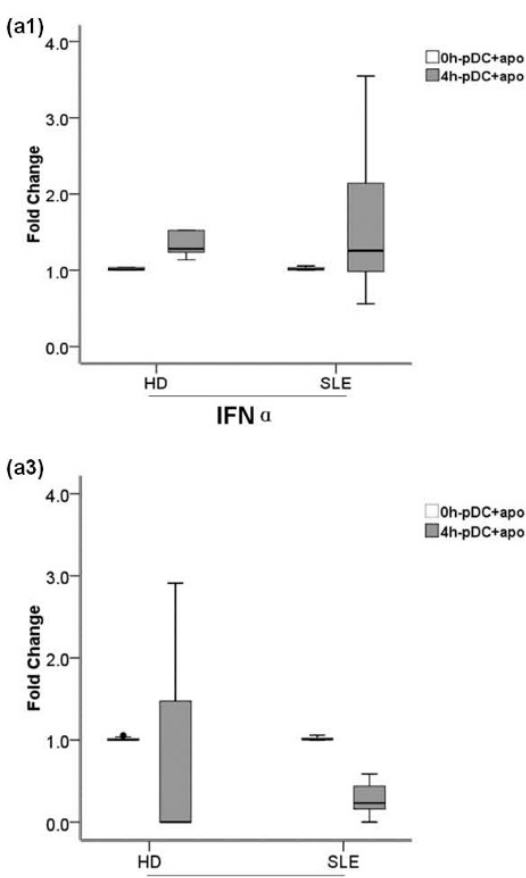

IL-10
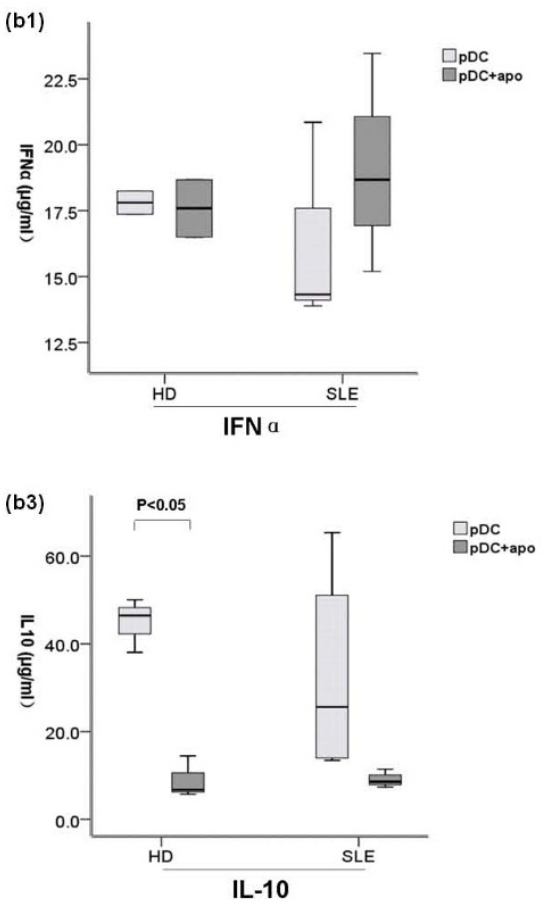
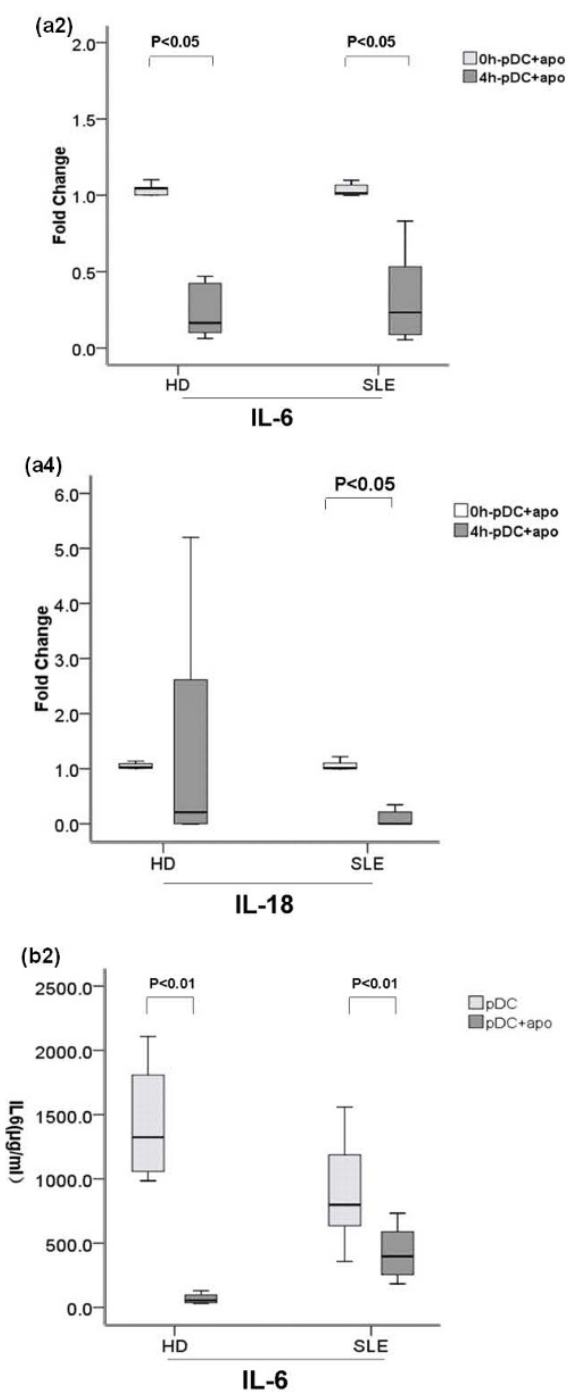

Figure 4 Changes in mRNA expression and protein levels of cytokines in HD and SLE pDCs + apoPMNs. (a) Changes in IFNa, IL-6, IL-10 and IL18 mRA expression in HD $(n=7)$ and SLE $(n=7) \mathrm{pDC}+$ + apoPMNs. IFNa mANA expression (A1) appeared increased in both HD and SLE pDCs + apoPMNs but the changes did not reach statistical significance. IL-6 (A2) mANA expression was significantly decreased in both HD and SLE pDCs + apoPMNs. IL-10 mRA expression (A3) did not change in both HD pDCs + apoPMNs. IL-18 mRA expression (A4) did not change in HD pDCs + apoPANs but was significantly decreased in SLE pDCs + apoPMNs. (b) protein levels of IFNa, IL-6 and IL-10 in the supernatants of cultured HD ( $n=8)$ and StE $(n=8)$ pDCs + apoPMNs (B1, B2 and B3). Results were compared with cultured pDCs alone. B1) No significant differences in the levels of IFNa in the culture supernatants of both HD and SLE pDCs + apoPMNs were detected. B2) Levels of IL- 6 were significantly decreased in the culture supernaants of both HD and SLE DC + apoPMNs. B3) Levels of IL-10 in the culture supernatants of HD pDCs + apoPMNs were significantly decreased while no significant changes were found in that of SLE DC + apoPMNs. Values are expressed in Median (interquartile range, range). pDCs + apoPMNs, pDCs that had been loaded with apoptotic polymorphonuclear cells; HD, healthy donors; INa, Interferon a; IL, Interleukin. 


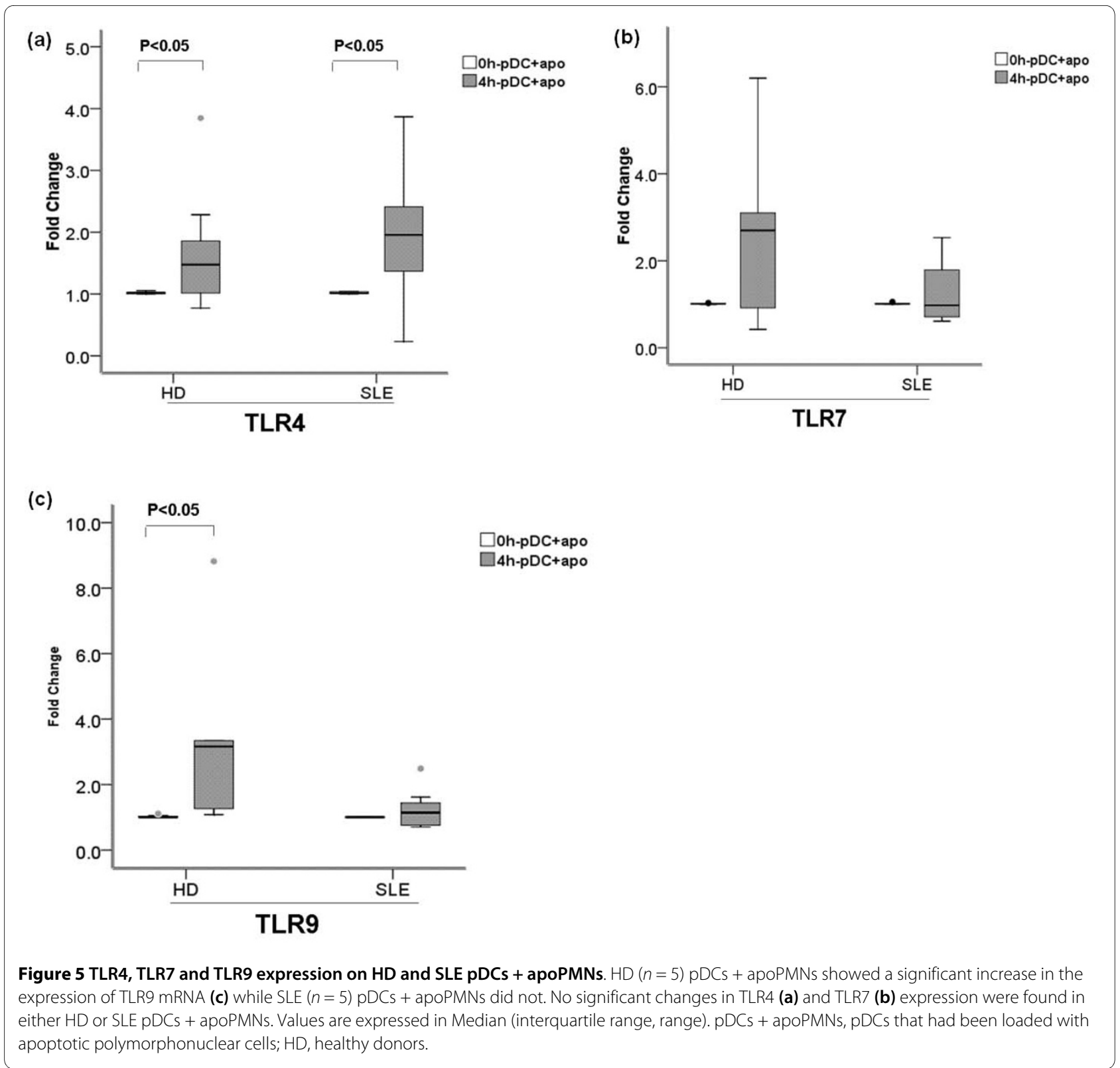

other studies have also suggested that an increased number of apoptotic cells in the circulating blood are associated with autoimmunity $[10,24]$. Thus, we designed the current series of experiments to investigate the capacity of apoptotic cell loaded pDCs in stimulating $\mathrm{T}$ cells. To our knowledge, this is the first study which evaluated the effects of directly isolated peripheral pDCs on T cells in healthy subjects and patients with SLE. Our results show that healthy pDCs + apoPMNs did not induce autologous $\mathrm{T}$ cell proliferation (Figure 1a). This lack of ability of pDCs to stimulate self $\mathrm{T}$ cells even when they encountered apoptotic cells suggests that pDCs may play a tolerance role in immunity in health. Indeed, previous animal studies have also suggested a tolerance role of pDCs [25].
In humans, pDCs have been found to elicit antigen-specific anergy in $\mathrm{CD}^{+} \mathrm{T}$ cell lines [26]. Furthermore, monocty-derived DCs were reported to be poor stimulators of T cells [25]. Our previous study on human circulating pDCs also suggested a low stimulatory capacity of pDCs as evidenced by their low level of co-stimulatory surface molecule expression [18].

We also investigated the ability of pDCs to stimulate allogenous $\mathrm{T}$ cells as an assessment of the antigen-presenting capability of DCs [27]. We found that healthy pDCs + apoPMNs induced only a very low level of allogenous $\mathrm{T}$ cell proliferation (Figure 1b) while SLE pDCs were more capable of inducing allogenous $\mathrm{T}$ cell proliferation (Figure 1b). Some previous studies have shown the oppo- 
site results. For example, DC-enriched APCs from SLE patients have been shown to have diminished T cell stimulatory capacity [28], or SLE peripheral DCs had reduced capacity to induce concanavalin A (Con A)-stimulated T cell proliferation [29]. However, these studies did not use purified DCs and their functions may be altered by other cells that were present in the assay.

In accordance with the proliferation results above, allogenous $\mathrm{CD}^{+}{ }^{+} \mathrm{T}$ cells that were co-cultured with either healthy or SLE pDCs + apoPMNs were found to have an increased expression of CD25 (Figure 2b). $\mathrm{CD} 4{ }^{+} \mathrm{CD} 25^{+} \mathrm{T}$ cells may consist of either specific Th or Treg cell subsets [21]. As Foxp3 is now regarded as the specific signature of Treg cells [22], we evaluated the expression of Foxp3 $3^{+}$in allogenous $\mathrm{CD} 4{ }^{+} \mathrm{CD} 25^{+} \mathrm{T}$ cells induced by pDCs, only healthy pDCs + apoPMNs were found to have increased Foxp3 expression (Figure 2d), indicating the development of Treg cells. This is consistent with a recent mouse model which showed that murine pDCs that had acquired alloantigens from allografts during transplant mediated antigen-specific Treg cell development and allograft tolerance [30]. Studies on human pDCs have also revealed that CpG-oligodeoxynucleotides (ODN) stimulated circulating pDCs were able to induce Treg cell expansion [31]. Treg cells maintain peripheral tolerance by suppressing the activation and population expansion of self-reactive T cells [32].

Contrary to healthy pDCs - apoPMNs, SLE pDCs + apoPMNs did not induce Foxp3 ${ }^{+}$expression in $\mathrm{CD} 4{ }^{+} \mathrm{CD} 25^{+} \mathrm{T}$ cells (Figure 2d). The important role of Foxp $3{ }^{+} \mathrm{CD} 4{ }^{+} \mathrm{CD} 25^{+}$Treg cells in immune homeostasis is that it controls autoimmunity throughout life. In animal models, Treg cell removal by neonatal thymectomy causes the spontaneous development of various organspecific autoimmune diseases $[33,34]$. Therefore, the lack of capacity of SLE pDCs to induce Treg development may contribute to the break down of immune tolerance and the development of autoimmunity. Indeed, SLE patients have been found to have significantly lower numbers of $\mathrm{CD} 4{ }^{+} \mathrm{CD} 25^{+} \mathrm{T}$ cells than normal persons [35]. In addition, a decreased suppressive function of $\mathrm{CD} 4+\mathrm{CD} 25^{\text {high }}$ Treg cells with reduced level of Foxp3 mRNA and protein expression has also been found in active SLE [36]. Recently, a study has shown that APCs from SLE patients were responsible for decreased Treg cell activity though the authors had used non-T cells as APCs [37].

An important feature that differentiates the various subsets of $\mathrm{T}$ cells is the characteristic profile of cytokines produced by these cells $[38,39]$. Our results showed that both healthy and SLE pDCs + apoPMNs stimulated T cells produced Th1 related IFNY and IL-2 (Figure 3a2, $3 \mathrm{~b} 2$ ). Only healthy pDCs + apoPMNs stimulated $\mathrm{T}$ cells produced Treg 2 cell-related TGF $\beta$ (Figure 3c) though the difference did not reach statistical significance. However, this latter finding provides further support of the notion that healthy pDCs + apoPMNs induce tolerogenic Treg cell expansion while SLE pDCs + apoPMNs do not.

Functional changes of pDCs + apoPMNs including cytokine production and TLR expression [40] were studied to delineate the probable mechanisms of these $\mathrm{pDCs}$ effects on $\mathrm{T}$ cells. We found that circulating healthy pDCs + apoPMNs had decreased mRNA expression and protein levels of IL-6 (Figure 4a2) and protein levels of IL-10 (Figure 4B3). SLE pDCs + apoPMNs were also found to have decreased mRNA expression and protein levels of IL-6 (Figure 4a2). However, we also noted a decrease in IL-18 mRNA expression (Figure 4a4) and no significant changes in IL-10 protein levels in the supernatant of the SLE pDCs + apoPMNs cultures. These findings are interesting as DC IL-18 is involved in the induction of Th1 response and IL-10 induces Th2 response during interactions with T-cells. Our findings are in accordance with the notion that SLE is characterized by a Th1/Th2 imbalance toward Th2 dominance.

TLRs play an important role in the presentation of antigens derived from apoptotic cells by DCs [40-43]. In our study, we found that healthy pDCs + apoPMNs increased their expression of TLR9 mRNA (Figure 5c) and TLR4 mRNA (Figure 5a) while TLR7 (Figure 5b) mRNA expression remained unchanged (Figure 5a). A previous study has reported that human pDCs activated by the TLR9 ligand $\mathrm{CpG}-\mathrm{ODN}$ could induce $\mathrm{CD} 4{ }^{+} \mathrm{CD} 25^{+}$Treg cells [31]. With SLE pDCs + apoPMNs, however, no increase in TLR9 mRNA expression was found (Figure 5c). This is consistent with data presented in a previous study which found that the expression of TLR9 on SLE pDCs stimulated by CpG ODN decreased rapidly [44]. We hypothesize that this difference in TLR9 recruitment between SLE and healthy pDCs may partly contribute to the lack of induction of Treg cells development in SLE, and this may further contribute to the tolerance break-down and autoimmune disease development.

A limitation of this study is that most of the patients were receiving some form of treatment including immunosuppressive agents. It is therefore not possible to confirm whether the pDC changes were a result of the underlying disease or that of the various lupus medications. It should, however, be noted that differential changes were seen in patients with different levels of lupus disease activity. In general, the more active the disease was, the more adverse pDC changes were noted. It is therefore tempting to suggest that our findings reflect the true role of pDCs in lupus disease pathogenesis. Future studies should aim to recruit treatment of naïve or newly diagnosed patients with SLE. However, this will have to involve the collaboration of multiple lupus research units. It has taken the authors over two years to recruit 58 suit- 
able patients from a cohort of over 500 patients for the purpose of this study.

\section{Conclusions}

Our study has provided further insights into the role of pDCs in SLE. In patients with SLE, the capacity of circulating $\mathrm{pDCs}$ to stimulate $\mathrm{T}$ cells was increased while their ability to induce Treg cell development was decreased. These may be the results of decreased IL-18 and increased IL-10 transcription which may prime Th2 response, and low expression of TLR9 following pDCs' interaction with apoptotic cells.

\begin{abstract}
Abbreviations
AC-BMDCs: apoptotic cell-pulsed bone marrow-derived DCs; APCs: antigen presenting cells; DCs: dendritic cells; IFN: interferon; IL, interleukin; ODN: oligodeoxynucleotides; PBMCs: peripheral blood mononuclear cells; PCR: real time polymerase chair reaction; pDCs: plasmacytoid DCs; pDCs: + apoPMNs, pDCs: that had captured apoptotic PMNs; PMNs: polymorphonuclear cells; RBCs: red blood cells; SLE: systemic lupus erythematosus; SLEDAI: SLE disease activity index; TGF: transfer growth factor; TLR: toll like receptor; TNF: tumor necrosis factor; Treg: T regulatory; UV: ultraviolet.
\end{abstract}

\section{Competing interests}

The authors declare that they have no competing interests.

\section{Authors' contributions}

OJ carried out the whole study, performed the statistical analysis and drafted the manuscript. SK, RF and YN participated in some of the cell isolation and interaction assays. MYM participated in patient recruitment. LS and JG helped to draft the manuscript and performed the statistical analysis. AC carried out the flow cytometry, ELISA and MLR assays. JY participated in real time PCR detection. CSL conceived, participated in the design of and sought funding for the study. He coordinated patient recruitment and helped draft the manuscript. All authors read and approved the final manuscript.

\section{Acknowledgements}

This study was funded by the University Grants Council research grant and The Government of Hong Kong SAR. We thank Miss Ivy Law for her help in drawing blood and Dr. Li-wei Lu and Mr. Otis Ko for their technological suggestions. We thank Miss Helen Law, Dr. Yu-lung Lau and Dr. Eddie Ip for their kind help in the initial stage of setting up the experiments. Thanks are also due to Miss Jessie Wong for her secretarial help and all the subjects who took part in the experiments.

\section{Author Details}

'Division of Rheumatology and Clinical Immunology, Department of Medicine, Queen Mary Hospital, The University of Hong Kong, 102 Pokfulam Road, Hong Kong, PR China, 2Department of Rheumatology, Third Hospital of Yat-sen Sun University, 600 Tian He Road, Guang Zhou, 510630, PR China and ${ }^{3}$ Department of Rheumatology, Drum Tower Hospital, The University of Nanjing, 321 Zhong Shan Road, Nanjing, 210008, PR China

Received: 13 October 2009 Revised: 12 May 2010

Accepted: 9 July 2010 Published: 9 July 2010

\section{References}

1. Banchereau J, Briere F, Caux C, Davoust J, Lebecque S, Liu YJ, Pulendran B, Palucka K: Immunobiology of dendritic cells. Annu Rev Immunol 2000, 18:767-811

2. Palucka AK, Banchereau J, Blanco P, Pascual V: The interplay of dendritic cell subsets in systemic lupus erythematosus. Immunol Cell Biol 2002, 80:484-488.

3. Doganci A, Eigenbrod T, Krug N, De Sanctis GT, Hausding M, Erpenbeck VJ, Haddad el B, Lehr HA, Schmitt E, Bopp T, Kallen KJ, Herz U, Schmitt S, Luft C, Hecht O, Hohlfeld JM, Ito H, Nishimoto N, Yoshizaki K, Kishimoto T, Rose-John S, Renz H, Neurath MF, Galle PR, Finotto S: The IL-6R alpha chain controls lung CD4 + CD25 + Treg development and function during allergic airway inflammation in vivo. J Clin Invest 2005, 115:313-325

4. Pasare C, Medzhitov R: Toll pathway-dependent blockade of CD4 + CD25 + T cell-mediated suppression by dendritic cells. Science 2003, 299:1033-1036.

5. Iwasaki A, Kelsall BL: Freshly isolated Peyer's patch, but not spleen, dendritic cells produce interleukin 10 and induce the differentiation of Thelper type 2 cells. J Exp Med 1999, 190:229-239.

6. Calvani N, Tucci M, Richards HB, Tartaglia P, Silvestris F: Th1 cytokines in the pathogenesis of lupus nephritis: the role of IL-18. Autoimmun Rev 2005, 4:542-548.

7. Blanco P, Palucka AK, Gill M, Pascual V, Banchereau J: Induction of dendritic cell differentiation by IFN-alpha in systemic lupus erythematosus. Science 2001, 294:1540-1543.

8. Ronnblom L, Alm GV: Systemic lupus erythematosus and the type I interferon system. Arthritis Res Ther 2003, 5:68-75.

9. Blomberg S, Eloranta ML, Magnusson M, Alm GV, Ronnblom L: Expression of the markers BDCA-2 and BDCA-4 and production of interferonalpha by plasmacytoid dendritic cells in systemic lupus erythematosus. Arthritis Rheum 2003, 48:2524-2532.

10. Kim T, Kanayama Y, Negoro N, Okamura M, Takeda T, Inoue T: Serum levels of interferons in patients with systemic lupus erythematosus. Clin Exp Immunol 1987, 70:562-569.

11. Jin O, Sun LY, Zhou KX, Zhang XS, Feng XB, Mok MY, Lau CS: Lymphocyte apoptosis and macrophage function: correlation with disease activity in systemic lupus erythematosus. Clin Rheumatol 2005, 24:107-110.

12. Graham KLU, Paul J: Sources of autoantigens in systemic lupus erythematosus. Current Opinion in Rheumatology 2005, 17:513-517.

13. Boule MW, Broughton C, Mackay F, Akira S, Marshak-Rothstein A, Rifkin IR: Toll-like receptor 9-dependent and -independent dendritic cell activation by chromatin-immunoglobulin $\mathrm{G}$ complexes. J Exp Med 2004, 199:1631-1640.

14. Sato Y, Miyata M, Nishimaki T, Kochi H, Kasukawa R: CpG motifcontaining DNA fragments from sera of patients with systemic lupus erythematosus proliferate mononuclear cells in vitro. J Rheumatol 1999, 26:294-301

15. Tzeng TC, Suen JL, Chiang BL: Dendritic cells pulsed with apoptotic cells activate self-reactive T-cells of lupus mice both in vitro and in vivo. Rheumatology (Oxford) 2006, 45:1230-1237.

16. Tan EM, Cohen AS, Fries JF, Masi AT, McShane DJ, Rothfield NF, Schaller JG, Talal N, Winchester RJ: The 1982 revised criteria for the classification of systemic lupus erythematosus. Arthritis Rheum 1982, 25:1271-1277.

17. Bombardier C, Gladman DD, Urowitz MB, Caron D, Chang CH: Derivation of the SLEDAI. A disease activity index for lupus patients. The Committee on Prognosis Studies in SLE. Arthritis Rheum 1992, 35:630-640

18. Jin O, Kavikondala S, Sun L, Fu R, Mok MY, Chan A, Yeung J, Lau CS: Systemic lupus erythematosus patients have increased number of circulating plasmacytoid dendritic cells, but decreased myeloid dendritic cells with deficient CD83 expression. Lupus 2008, 17:654-662.

19. Boor PP, ljzermans JN, van der Molen RG, Binda R, Mancham S, Metselaar HJ, Kusters JG, de Jong E, Drexhage HA, Kwekkeboom J: Immunomagnetic selection of functional dendritic cells from human lymph nodes. Immunol Lett 2005, 99:162-168.

20. Grouard G, Rissoan MC, Filgueira L, Durand I, Banchereau J, Liu YJ: The enigmatic plasmacytoid T cells develop into dendritic cells with interleukin (IL)-3 and CD40-ligand. J Exp Med 1997, 185:1101-1111.

21. Hill JA, Benoist C, Mathis D: Treg cells: guardians for life. Nat Immunol 2007, 8:124-125.

22. Fontenot JD, Gavin MA, Rudensky AY: Foxp3 programs the development and function of CD4 + CD25 + regulatory T cells. Nat Immunol 2003 4:330-336.

23. Steinman RM, Turley S, Mellman I, Inaba K: The induction of tolerance by dendritic cells that have captured apoptotic cells. J Exp Med 2000, 191:411-416.

24. Frisoni L, McPhie L, Colonna L, Sriram U, Monestier M, Gallucci S, Caricchio R: Nuclear autoantigen translocation and autoantibody opsonization lead to increased dendritic cell phagocytosis and presentation of nuclear antigens: a novel pathogenic pathway for autoimmunity? Immunol 2005, 175:2692-2701. 
25. Abe M, Wang Z, de Creus A, Thomson AW: Plasmacytoid dendritic cell precursors induce allogeneic $\mathrm{t}$-cell hyporesponsiveness and prolong heart graft survival. American Journal of Transplantation 2005, 5:1808-1819.

26. Kuwana M, Kaburaki J, Wright TM, Kawakami Y, Ikeda Y: Induction of antigen-specific human CD4(+) T cell anergy by peripheral blood DC2 precursors. Eur J Immunol 2001, 31:2547-2557.

27. Steinman RM, Gutchinov B, Witmer MD, Nussenzweig MC: Dendritic cells are the principal stimulators of the primary mixed lymphocyte reaction in mice. J Exp Med 1983, 147:613-627.

28. Scheinecker C, Zwolfer B, Koller M, Manner G, Smolen JS: Alterations of dendritic cells in systemic lupus erythematosus: phenotypic and functional deficiencies. Arthritis Rheum 2001, 44:856-865.

29. Katagiri T: The dysfunction of human peripheral blood dendritic cells on concanavalin A-induced T cell responses in patients with systemic lupus erythematosus. Arerugi 1992, 41:693-698.

30. Ochando JC, Homma C, Yang Y, Hidalgo A, Garin A, Tacke F, Angeli V, Li Y, Boros P, Ding Y, Jessberger R, Trinchieri G, Lira SA, Randolph GJ, Bromberg JS: Alloantigen-presenting plasmacytoid dendritic cells mediate tolerance to vascularized grafts. Nature Immunology 2006, 7:652-662.

31. Moseman EA, Liang X, Dawson AJ, Panoskaltsis-Mortari A, Krieg AM, Liu YJ, Blazar BR, Chen W: Human plasmacytoid dendritic cells activated by $\mathrm{CpG}$ oligodeoxynucleotides induce the generation of CD4+CD25 + regulatory T cells. J Immunol 2004, 173:4433-4442.

32. Sakaguchi S: Naturally arising Foxp3-expressing CD25 + CD4 + regulatory $\mathrm{T}$ cells in immunological tolerance to self and non-self. $\mathrm{Nat}$ Immunol 2005, 6:345-352.

33. O'Garra A, Vieira P: Twenty-first century Foxp3. Nat Immunol 2003, 4:304-306.

34. Maloy KJ, Powrie F: Regulatory T cells in the control of immune pathology. Nat Immunol 2001, 2:816-822.

35. Liu MF, Wang CR, Fung LL, Wu CR: Decreased CD4 + CD25 + T cells in peripheral blood of patients with systemic lupus erythematosus. Scand J Immunol 2004, 59:198-202.

36. Valencia X, Yarboro C, Illei G, Lipsky PE: Deficient CD4 + CD25high T regulatory cell function in patients with active systemic lupus erythematosus. J/mmunol 2007, 178:2579-2588.

37. Yan B, Ye S, Chen G, Kuang M, Shen N, Chen S: Dysfunctional CD4 +,CD25 + regulatory $T$ cells in untreated active systemic lupus erythematosus secondary to interferon-alpha-producing antigen-presenting cells. Arthritis Rheum 2008, 58:801-812.

38. Groux H, O'Garra A, Bigler M, Rouleau M, Antonenko S, de Vries JE, Roncarolo MG: A CD4 + T-cell subset inhibits antigen-specific T-cell responses and prevents colitis. Nature 1997, 389:737-742.

39. Weiner $\mathrm{HL}$ : Oral tolerance: immune mechanisms and the generation of Th3-type TGF-beta-secreting regulatory cells. Microbes Infect 2001, 3:947-954.

40. Krug A, Towarowski A, Britsch S, Rothenfusser S, Hornung V, Bals R, Giese T, Engelmann H, Endres S, Krieg AM, Hartmann G: Toll-like receptor expression reveals CpG DNA as a unique microbial stimulus for plasmacytoid dendritic cells which synergizes with CD40 ligand to induce high amounts of IL-12. Eur J Immunol 2001, 31:3026-3037.

41. Takeda K, Kaisho T, Akira S: Toll-like receptors. Annu Rev Immunol 2003, 21:335-376

42. Jin O, Sun L, Kavikondala S, Lau C: Innate immunity and systemic lupus erythematosus. APLAR Journal of Rheumatology 2006, 9:359-364.

43. Means TK, Latz E, Hayashi F, Murali MR, Golenbock DT, Luster AD: Human lupus autoantibody-DNA complexes activate DCs through cooperation of CD32 and TLR9. J Clin Invest 2005, 115:407-417.

44. Hornung V, Rothenfusser S, Britsch S, Krug A, Jahrsdorfer B, Giese T, Endres S, Hartmann G: Quantitative expression of toll-like receptor 1-10 mRNA in cellular subsets of human peripheral blood mononuclear cells and sensitivity to CpG oligodeoxynucleotides. J Immunol 2002, 168:4531-4537.

\section{doi: 10.1186/ar3075}

Cite this article as: Jin et al, Abnormalities in circulating plasmacytoid dendritic cells in patients with systemic lupus erythematosus Arthritis Research \& Therapy 2010, 12:R137

\section{Submit your next manuscript to BioMed Centra and take full advantage of:}

- Convenient online submission

- Thorough peer review

- No space constraints or color figure charges

- Immediate publication on acceptance

- Inclusion in PubMed, CAS, Scopus and Google Scholar

- Research which is freely available for redistribution 